\title{
Calculation of Crystallographic Texture due to Displacive Transformations
}

\author{
H. K. D. H. Bhadeshia ${ }^{\dagger}$, H. Abreu ${ }^{\dagger \ddagger}$ and S. Kundu* \\ International Journal of Materials Research, 99 (2008) 342-346 \\ $\ddagger$ University of Cambridge, Materials Science and Metallurgy, Cambridge, U.K. \\ ${ }^{\ddagger}$ Universidade Federal do Ceará, Engenharia Metalúrgica e de Materiais, Fortaleza, Brasil \\ *TATA Steel, Research and Development Division, Jamshedpur, India
}

\begin{abstract}
Displacive transformations involve the disciplined motion of atoms. As a result, there are clearly defined relationships between all aspects of the parent and product lattices. The theory for this is well established but has not been exploited in the calculation of transformation textures. This paper is a critical assessment of the methods for the estimation of crystallographic textures during the displacive transformation of austenite into martensite, bainite or Widmanstätten ferrite in steels. The discussion is limited to the case where austenite is not in a plastically deformed state prior to its transformation.
\end{abstract}

Keywords: texture, martensite, bainite, variant selection, displacive transformations

\section{Introduction}

${ }^{1}$ Crystallographic texture is said to exist in a polycrystalline material when the distribution of crystal orientations is not random relative to a frame of reference. An understanding of texture can help relate single-crystal properties to those of aggregates of crystals [1,2]. The texture can also be used to engineer the properties of grain boundaries [3].

\footnotetext{
${ }^{1}$ This paper is a part of a special issue published in honour of Professor Horst Cerjak who has had a life-long and deep interest in metallurgy. As a gentleman and a scholar, he finds the time to maintain close relationships with colleagues all over the world and has been a major driving force in the subject. The work presented here is inspired by the emerging field of texture-controlled weld metals, as a means for counteracting the development of residual stresses in welds. Welding is of course Professor Cerjak's favourite field and we hope that he appreciates the contents of this paper.
} 
A material can become textured due to grain rotation during deformation; thus in hexagonal closepacked zirconium, the basal planes of the grains tend to align with the rolling plane during the production of sheet [4]. Selective phenomena during recovery, recrystallisation and grain growth can change the character of the final texture [5,6]. Epitaxy during deposition obviously can lead to textures in coatings $[7,8]$ as can heat-treatment in an electrical or magnetic field [9].

In solid-state phase transitions, the transformation texture can be calculated from a knowledge of the orientations of the parent crystals and their orientation relationship with the product phase [10]. This assumes that the product phase grows in the parent crystal in which it nucleates. In a displacive transformation, the crystal structure of the parent is deformed into that of the product without the need for any diffusion. Since the co-ordinated movements of atoms associated with this transformation mechanism cannot in general be sustained across grain boundaries, it is reasonable to assume that the product is confined to the parent grain with which it has an orientation relationship. The purpose of this paper is to focus on displacive transformations with a view to assessing the variety of methods used in estimating transformation textures in steels, although the issues of importance are generic. Given the page limits set by the organisers of this special issue, we limit ourselves to austenite which is not plastically deformed prior to its transformation.

Crucial to the calculation is the nature of the orientation relationship; we begin therefore with an explanation of the origin of this relationship, in the context of martensitic transformations; the general theory is applicable also to bainite and Widmanstätten ferrite.

\section{Crystallographic Theory: Orientation Relationships}

Consider the displacive transformation of austenite ( $\gamma$, cubic-close packed crystal structure) to martensite $\left(\alpha^{\prime}\right.$, body-centred cubic or body-centred tetragonal). The change in crystal structure is achieved by a homogeneous deformation known as the Bain strain $\mathbf{B}$, which although proposed in 1924 [11], has stood the test of time as the pure deformation which achieves the desired change with the smallest strains [12]. The diagonal terms of $\mathbf{B}$ are given by $a_{\alpha^{\prime}} / a_{\gamma}, \sqrt{2} a_{\alpha^{\prime}} / a_{\gamma}$ and $\sqrt{2} a_{\alpha^{\prime}} / a_{\gamma}$, whereas the remaining terms are zero when $\mathbf{B}$ is defined relative to the principal axes. $a_{\alpha^{\prime}}$ and $a_{\gamma}$ are the lattice parameters of martensite and austenite, respectively. The Bain correspondence is illustrated in Fig. 1 and implies the orientation relationship:

$$
\left[\begin{array}{lll}
0 & 0 & 1
\end{array}\right]_{\gamma}\left\|\left[\begin{array}{lll}
0 & 0 & 1
\end{array}\right]_{\alpha^{\prime}} \quad\left[\begin{array}{lll}
1 & \overline{1} & 0
\end{array}\right]_{\gamma}\right\|\left[\begin{array}{lll}
1 & 0 & 0
\end{array}\right]_{\alpha^{\prime}} \quad\left[\begin{array}{lll}
1 & 1 & 0
\end{array}\right]_{\gamma} \|\left[\begin{array}{lll}
0 & 1 & 0
\end{array}\right]_{\alpha^{\prime}}
$$

This orientation is not observed experimentally because the strain energy associated with $\mathbf{B}$ would be too large, several $\mathrm{kJ} \mathrm{mol}^{-1}$ [13], which is far in excess of the chemical driving force for transformation [14].

Furthermore, the Bain strain does not satisfy the minimum requirement of martensitic transformation, that the deformation must leave one line invariant in order to ensure sufficient coherency in the $\gamma / \alpha^{\prime}$ interface to enable it to move without diffusion [15-19]. This can be seen in Figs. 2a, b; the austenite is represented as a sphere which, as a result of the Bain strain $\mathbf{B}$, is deformed into an ellipsoid of revolution which represents the martensite. There are no lines which are left undistorted or unrotated by $\mathbf{B}$. There are no lines in the $\left(\begin{array}{llll}0 & 0 & 1\end{array}\right)_{\gamma}$ plane which are undistorted. The lines 
$a b$ and $c d$ are undistorted but are rotated to the new positions $a^{\prime} b^{\prime}$ and $c^{\prime} d^{\prime}$. Such rotated lines are not invariant. However, the combined effect of the Bain strain $\mathbf{B}$ and the rigid body rotation $\mathbf{R}$ is indeed an invariant-line strain (ILS) because it brings $c d$ and $c^{\prime} d^{\prime}$ into coincidence (Fig. 2c). This is the reason why the observed irrational orientation relationship differs from that implied by the Bain strain. Indeed, the rotation required to convert $\mathbf{B}$ into an invariant line strain precisely corrects the Bain orientation into that which is observed experimentally.

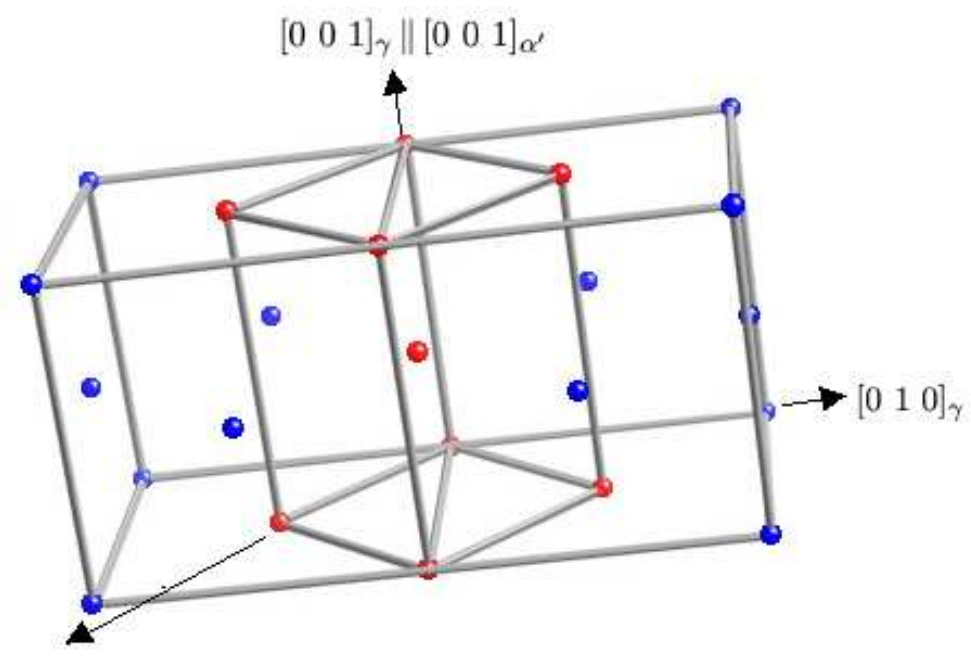

$\left[\begin{array}{lll}1 & \overline{1} & 0\end{array}\right]_{\gamma} \|\left[\begin{array}{lll}1 & 0 & 0\end{array}\right]_{\alpha^{\prime}}$

Figure 1: Two face-centred cubic unit cells of austenite, together with a body-centred tetragonal cell of austenite. The Bain strain (not illustrated here) involves a compression of the body-centred tetragonal cell of austenite along $[001]_{\gamma}$ and a uniform expansion on the $(001)_{\gamma}$ plane.

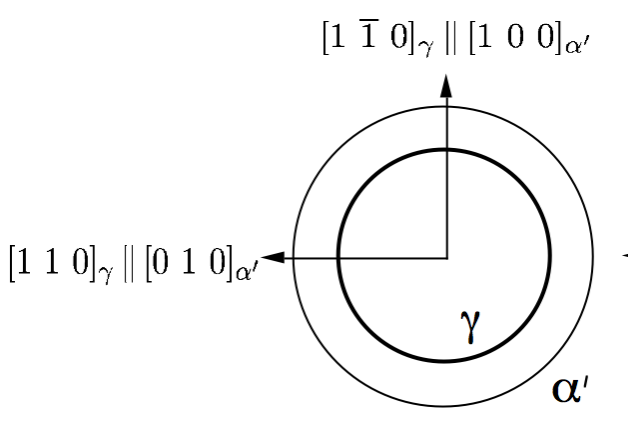

(a)

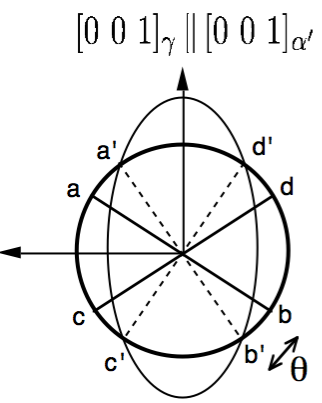

(b)

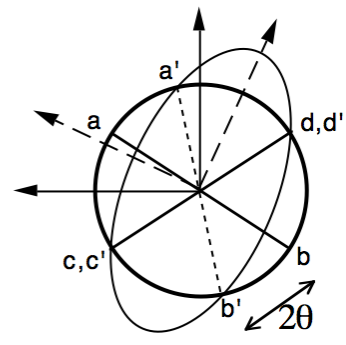

(c)

Figure 2: (a) and (b) show the effect of the Bain strain on austenite, which when undeformed is represented as a sphere of diameter $a b=c d$ in three-dimensions. The strain transforms it to an ellipsoid of revolution. (c) shows the ILS obtained by combining the Bain strain with a rigid body rotation through an angle $\theta$. 
It is now possible to reach some conclusions regarding orientation relationships in relation to transformation textures, whether these are for displacive or reconstructive phase change [20,21].

- The Bain orientation relationship does not exist. It is not appropriate to use this in calculating transformation texture. Any favourable conclusions reached based on the Bain orientation $[22,23]$ must be regarded as fortuitous [20].

- The Bain deformation is an incomplete description of the transformation strain and hence should not form the basis for variant selection (e.g. [24]).

As stated above, the combination $\mathbf{B R}$ predicts the exact orientation relationship which is irrational. However, it is often assumed in texture analysis that the orientation relationship between the austenite and martensite is that due to Kurdjumov-Sachs (KS) or Nishiyama-Wasserman (NW) $[22,25-27]$, but it has been known for some time that the true relation must be irrational [1518]. Although the difference between this irrational and assumed orientation may seem less than a few degrees, it is vital because the assumed orientations do not in general lead to an invariantline between the parent and product lattices. The existence of an invariant line is an essential requirement for martensitic transformation to occur. It is not surprising therefore, that Nolze [28] in his experimental study of several hundred thousand $\gamma / \alpha$ orientation relations, found detailed deviations from assumed Kurdjumov-Sachs etc. orientations.

Much is often made of the fact that there are 24 variants of KS and only 12 of NW. However, if the actual irrational orientation is used then there will always be 24 variants. As far as the authors are aware, there are no reported examples of only twelve variants of martensite per austenite grain.

The kind of orientation which emerges from the application of $\mathbf{B R}$ is listed in Table 1 [18, 21, 29, 30] where $\left(\gamma \mathrm{J} \alpha^{\prime}\right)$ represents the orientation relationship, and it is seen from the lower half of the table that neither the closely-packed planes nor the close-packed directions are parallel, as would be expected from a NW or KS orientation in which $\{111\}_{\gamma} \|\{011\}_{\alpha^{\prime}}$ and $\phi=0$ or $5.26^{\circ}$, where $\phi$ is the angle between $<10 \overline{1}>_{\gamma}$ and $<1 \overline{1} 1>_{\alpha^{\prime}}$.

Table 1: Typical crystallographic set for martensitic transformation for $a_{\gamma} / a_{\alpha^{\prime}}=1.2557$.

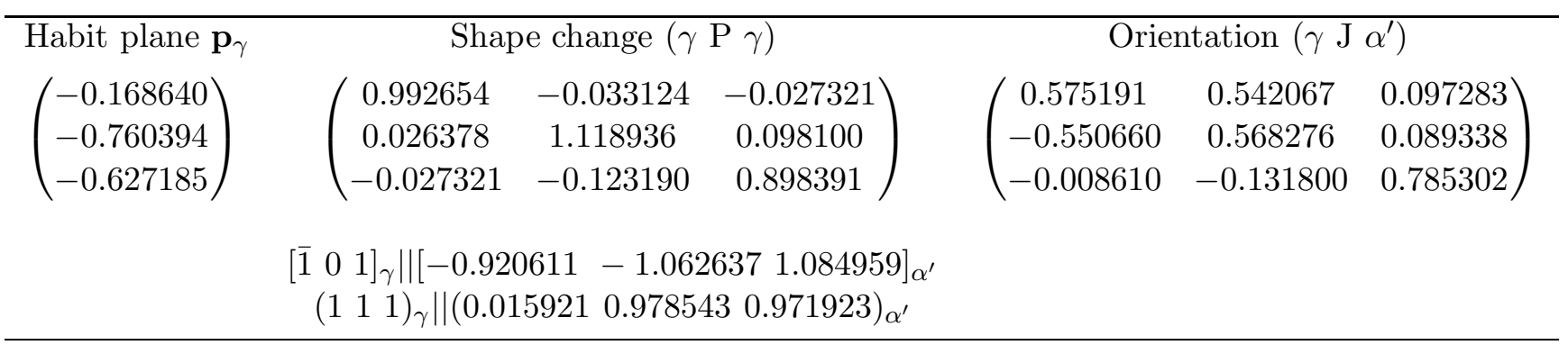

Suppose that it is assumed that $\{111\}_{\gamma} \|\{011\}_{\alpha^{\prime}}$, then it is only possible to satisfy the minimum condition for martensitic transformation at special values of the lattice parameter ratio $a_{\gamma} / a_{\alpha^{\prime}}[31]$, Fig. 3. 


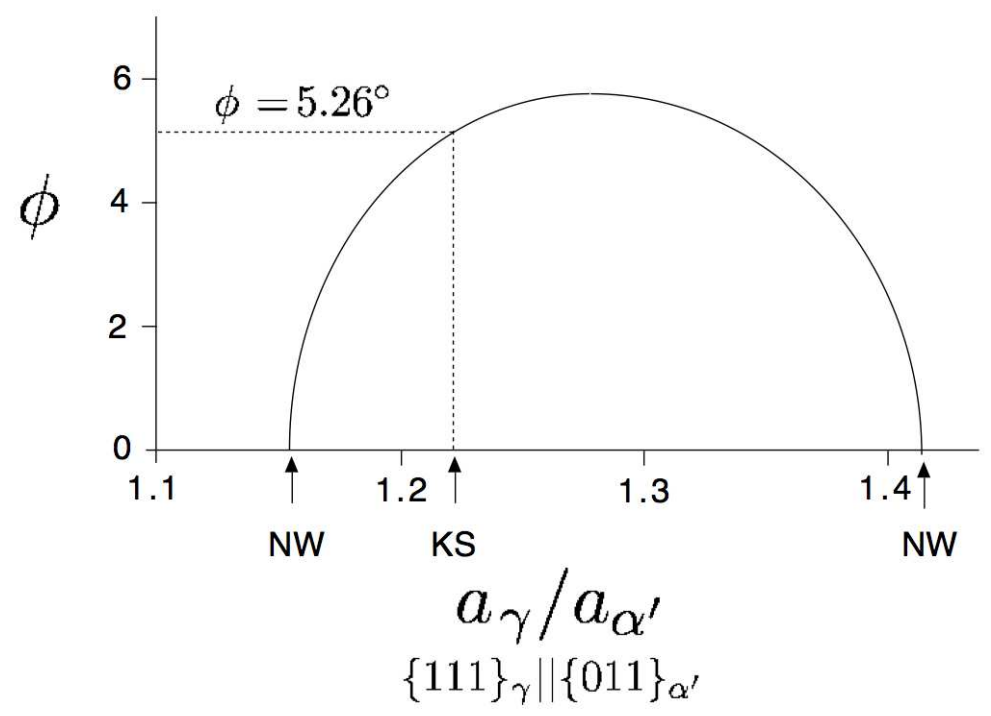

Figure 3: The angle $\phi$ must be such as to lie on the curve in order to ensure an invariant line [31]. KS occurs at $a_{\gamma} / a_{\alpha^{\prime}}=1.2247$.

\section{The Crystallographic Set}

The full theory of martensite [15-17] is beyond the scope of this paper; detailed descriptions and reviews can be found elsewhere [18, 32]. However, the development of transformation texture requires more than just the orientation relationship because not all possible variants may form depending on the interaction of the martensite with the imposed stresses and strains.

Displacive transformations are best regarded as deformation mechanisms which at the same time alter the crystal structure. The variant selection problem then reduces to issues similar to the selection of slip systems out of all the possibilities available during single-crystal deformation [1]. A slip system consists of a slip plane and slip direction, for example, (111)[10 $\overline{1}]$ is one of 12 crystallographically equivalent systems in austenite. An applied stress is resolved on to each of the slip systems, and that which has the highest resolved shear stress is said to be activated.

By analogy, the deformation due to martensitic transformation occurs on the habit plane (unit normal $\mathbf{p}$ ) in a displacement direction (unit vector $\mathbf{d}$ ). Note however that the latter will not lie precisely in the habit plane because the dilatational strain due to the volume change of transformation is directed normal to the habit plane. The dominating strain is the shear parallel to the habit plane at about 0.26 . The total deformation is expressed as a $3 \times 3$ matrix $\mathbf{P}$ :

$$
(\gamma \mathrm{P} \gamma)=\mathrm{I}+m[\gamma ; \mathbf{d}]\left(\mathbf{p} ; \gamma^{*}\right)
$$

where $m$ is the magnitude of the shape deformation and $\gamma$ and $\gamma^{*}$ represent the real and reciprocal bases of the austenite. ${ }^{2}$ The matrix $\mathbf{P}$ thus completely defines the deformation system, and there will in general be 24 different variants.

\footnotetext{
${ }^{2}$ Throughout this paper we use the vector and matrix notation due to Bowles and MacKenzie which is particularly good at avoiding confusion between frames of reference $[15,18]$
} 
It is most important to realise that the Bain strain, rigid body rotation, and the shape deformation are all mathematically related through the phenomenological theory of martensite:

$$
\mathbf{B R}=\mathbf{P P}^{\prime}
$$

where $\mathbf{P}^{\prime}$ is a shear whose macroscopic consequence is cancelled by the lattice-invariant deformation. The shape deformation $\mathbf{P}$ is an invariant-plane strain and is the experimentally observed permanent shape change caused when martensite forms (see Fig. 21.3 of [32]). Equation 2 emphasises that the orientation relationship and shape deformation are mathematically related. It is not rigourous to assign an orientation and then use independent data for the shape deformation, as is sometimes done $[20,27,33]$. Similarly, the use of shears which are consistent with an assumed orientation relationship but not with the habit plane [34] contradicts the need for a self-consistent mathematical set.

When calculating the favoured system during slip deformation, it is the macroscopic shear on the slip plane and slip direction which determines selection through interaction with the applied stress. The detailed atomic motions (e.g. in surmounting Peierls barriers) or microscopic heterogeneities (due to the discrete nature of atoms) are irrelevant in the selection of the system. In a similar way, it is the interaction of the applied stress with $\mathbf{P}$ which determines variant selection. $\mathbf{B}$ and $\mathbf{R}$ or other factorisations of the shape deformation are incomplete descriptions of the relevant strain. The interaction energy which provides the mechanical driving force for transformation [35]:

$$
U=\sigma_{N} \zeta+\tau s
$$

where $\sigma_{N}$ is the stress component normal to the habit plane, $\tau$ is the shear stress resolved on the habit plane in the direction of shear and $\zeta$ and $s$ are the respective normal and shear strains associated with transformation. The energy $U$ can be used as a rigourous variant selection criterion when the stresses applied are less than those required to cause plasticity in the austenite prior to its transformation [30].

The conclusions that can be reached from the discussion in this section are:

- In calculating transformation texture is is necessary to use a a self-consistent crystallographic set, rather than make independent assumptions about the orientation relationship and shape deformation as is sometimes done. The set must be such that the lattice deformation $\mathbf{B R}$ is an invariant-line strain; the analysis in [33] does not satisfy this criterion.

- The deformation due to martensitic transformation is an invariant-plane strain $\mathbf{P}$. It is this which should be used to calculate the interaction energy (variant selection) rather than, for example, the Bain strain [36].

\section{Transformation Plasticity}

Consider an arbitrary vector $\mathbf{u}$ traversing a grain of austenite prior to transformation, as illustrated in Fig. 4a. This vector makes an intercept $\Delta \mathbf{u}$ with a domain of austenite that eventually ends up 


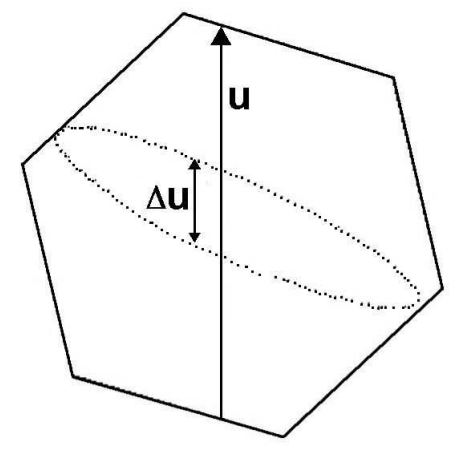

(a)

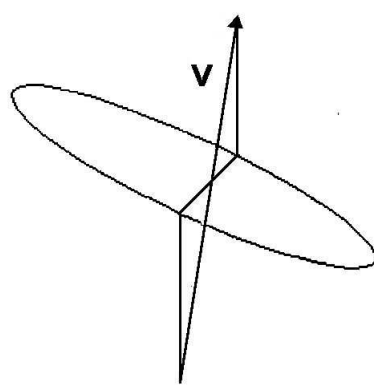

(b)

Figure 4: The deformation of an initial vector $\mathbf{u}$ by the formation of bainite. (a) An austenite grain prior to transformation, with the ultimate location of a plate of bainite marked. (b) Following displacive transformation. [30]

as a plate of bainite. As a consequence of the transformation, the vector $\mathbf{u}$ becomes a new vector $\mathbf{v}$ given by:

$$
\mathbf{v}=\mathbf{P} \Delta \mathbf{u}+(\mathbf{u}-\Delta \mathbf{u})
$$

When considering the formation of large number of bainite plates in many austenite grains, $\mathbf{u}$ traverses a polycrystalline sample of austenite so this equation must be generalised as follows:

$$
\mathbf{v}=\sum_{k=1}^{n} \sum_{j=1}^{24} \mathbf{P}_{j}^{k} \Delta \mathbf{u}_{j}^{k}+\left(\mathbf{u}-\sum_{k=1}^{n} \sum_{j=1}^{24} \Delta \mathbf{u}_{j}^{k}\right)
$$

where $j=1 \ldots 24$ represents the 24 crystallographic variants possible in each austenite grain, and $k=1 \ldots n$ represents the $n$ austenite grains traversed by the vector $\mathbf{u}$. In this scenario of a large number of bainite plates, the intercepts $\Delta \mathbf{u}_{j}^{k}$ can be approximated by $f_{j}^{k} \mathbf{u}$ where $f_{j}^{k}$ is the fraction of sample transformed by variant $j$ in austenite grain $k$.

The deformation caused by a particular plate $j$ in austenite grain $k$, i.e., $\left(\gamma_{k} \mathrm{P}_{\mathrm{j}} \gamma_{k}\right) \equiv \mathbf{P}_{j}^{k}$. The remaining 23 such matrices for grain 1 of austenite can be deduced from this using symmetry operations. They can then be expressed in the reference frame of the sample using a similarity transformation as follows:

$$
\left(\mathrm{S} \mathrm{P} \mathrm{P}_{j}^{k} \mathrm{~S}\right)=\left(\mathrm{S} \mathrm{R} \gamma_{k}\right)\left(\gamma_{k} \mathrm{P}_{j} \gamma_{k}\right)\left(\gamma_{k} \mathrm{R} \mathrm{S}\right)
$$

where $\left(\mathrm{S} \mathrm{R} \gamma_{k}\right.$ ) is the rotation matrix relating the basis vectors of the $k$ th austenite grain to the sample axes, and $\left(\gamma_{k} \mathrm{R} \mathrm{S}\right)$ is the inverse of that rotation matrix. In this way, the calculation described in equation 4 can be conducted in the sample frame of reference.

Some calculations illustrating the anisotropy of strains as a function of the number of crystallographic variants of martensite allowed are illustrated in Fig 5 for uniaxial tension and compression. That displacive transformations produce highly anisotropic strains when variant selection is significant has been demonstrated experimentally [37-39]. 

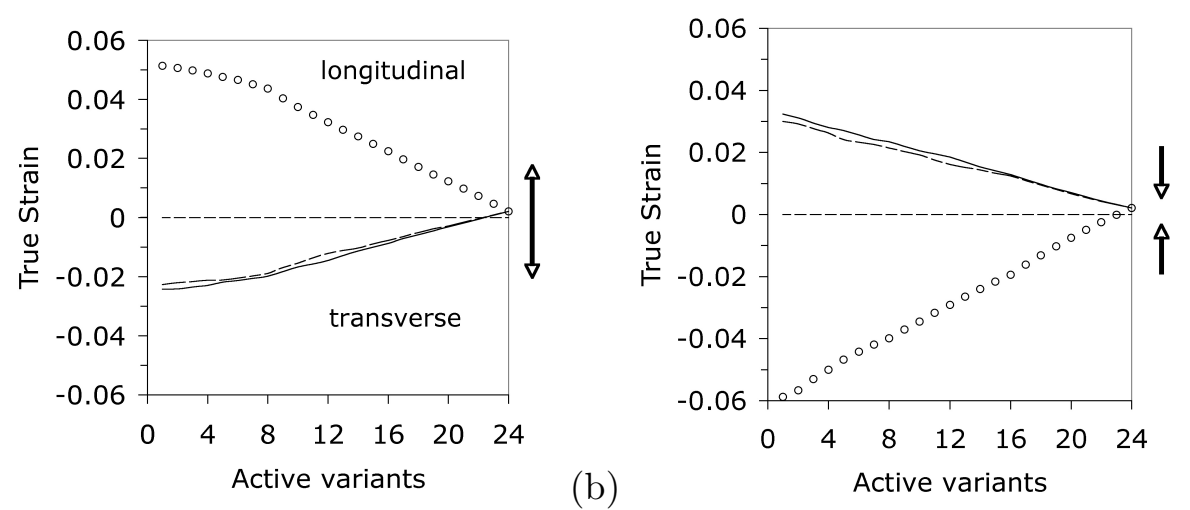

Figure 5: Strains developed due to transformation along the $\left[\begin{array}{lll}1 & 0 & 0\end{array}\right]_{S}$ direction (labelled longitudinal, along the stress axis), and the transverse directions $\left[\begin{array}{lll}0 & 1 & 0\end{array}\right]_{S}$ and $\left[\begin{array}{lll}0 & 0 & 1\end{array}\right]_{S}$. (a) Tensile stress. (b) Compressive stress.

- An important outcome of the fact that transformation strains can be calculated using the crystallographic set of martensite is that such strains can be exploited as an alternative or supplemental method of assessing texture.

\section{An Unresolved Issue}

The analysis of texture as described above and in the published literature, leaves open the question of the degree of variant selection as a function of the magnitude of the applied stress.

The total free energy available for transformation is the sum of chemical and mechanical components, the latter being zero in the absence of an applied stress during transformation [39]:

$$
\Delta G=\Delta G_{C H E M}+\Delta G_{M E C H}
$$

where $\Delta G_{M E C H} \equiv U$. It would be reasonable to assume that there is strong variant selection when the ratio of $\Delta G_{M E C H} / \Delta G$ is large [40]. This turns out to be the case as illustrated in Fig. 6 . There is a strong, albeit empirical, linear correlation between the ratio $\Delta G_{M E C H} / \Delta G$ and the number of most favoured variants allowed to form in each of the austenite grains [30]. This is an important observation in that it allows the extent of variant selection, and hence the transformation strains, to be calculated as a function of stress for any steel as long as the thermodynamic quantities can be estimated. Nevertheless, this clearly is an area where progress is needed from a fundamental point of view. An additional point to emerge from this analysis is the way in which the energy $U$ is calculated. Most publications treat the problem using elasticity theory with $U=\frac{1}{2} \sigma_{i j} \epsilon_{i j}$ but the strain due to transformation is plastic so the factor of $\frac{1}{2}$ should not be there. This only is important when using equation 7 because it becomes necessary to compare the relative magnitudes of the chemical and mechanical driving forces [41]. 


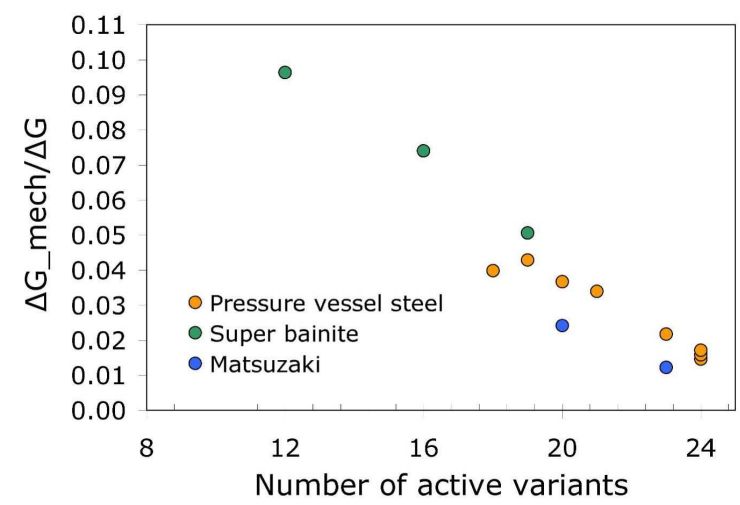

Figure 6: $\Delta G_{M E C H} / \Delta G$ versus the number of most favoured variants per grain $(n)$, for a variety of steels. [30].

\section{Summary}

This paper is limited to a discussion of transformation texture in steels where the austenite is not plastically deformed prior to its transformation.

It is clear that the calculation of the texture resulting from displacive transformation is best treated using the crystallographic theory of martensitic transformation. Furthermore, the strain relevant in calculating variant selection is the shape deformation due to martensite.

Whereas the current methods allow the orientations of crystals resulting from variant selection to be predicted, they do not permit their volume fractions to be estimated.

\section{References}

[1] E. Schmid, W. Boas: Plasticity of Crystals (translated from the 1935 edition of Kristalplastizitaet): F. A. Hughes and Co., London, U.K., 1950.

[2] D. Dorner, S. Zaefferer, L. Lahn, D. Raabe: Journal of Magnetism and Magnetic Materials 304 (2006) 183-186.

[3] L. Fionova, T. Watanabe, Y. Lisovski: ISIJ International 36 (1996) 613-623.

[4] B. A. Cheadle, S. A. Aldridge, C. E. Ells: Journal of Nuclear Materials 34 (1970) 119-124.

[5] F. J. Humphreys: Materials Science and Technology 15 (1999) 37-44.

[6] H. K. D. H. Bhadeshia: Materials Science and Engineering A A223 (1997) 64-77.

[7] I. Kanno, S. Fujii, T. Kamada, R. Takayama: Applied Physics Letters 70 (1997) 1378-1380.

[8] B. A. Glowacki, M. E. Vickers, N. A. Rutter, E. Maher, F. Pasotti, A. Baldini, R. Major: Journal of Materials Science 37 (2002) 157-168. 
[9] Z. G. Ye, A. M. Janner, H. Schmid: Journal of Physics - Condensed Matter 9 (1997) 26072621.

[10] H. J. Bunge, M. Humbert, P. I. Welch: Scripta Metallurgica 17 (1983) 1403-1405.

[11] E. C. Bain: Trans. AIME 70 (1924) 25-46.

[12] J. S. Bowles, C. M. Wayman: Metallurgical Transactions 3 (1972) 1113-1121.

[13] M. Suezawa: Materials Science and Engineering 48 (1981) 255-260.

[14] J. W. Christian: Thermodynamics and kinetics of martensite: in: G. B. Olson, M. Cohen (Eds.), International Conference on Martensitic Transformations ICOMAT '79: 1979: pp. $220-234$.

[15] J. S. Bowles, J. K. MacKenzie: Acta Metallurgica 2 (1954) 129-137.

[16] J. K. Mackenzie, J. S. Bowles: Acta Metallurgica 2 (1954) 138-147.

[17] M. S. Wechsler, D. S. Lieberman, T. A. Read: Trans. AIME Journal of Metals 197 (1953) 1503-1515.

[18] H. K. D. H. Bhadeshia: Geometry of Crystals: 2nd edition, Institute of Materials, 2001.

[19] J. W. Christian: Metallurgical Transactions A 13 (1982) 509-538.

[20] W. B. Hutchinson, L. Ryde, P. S. Bate: Materials Science Forum 495-497 (2005) 1141-1149.

[21] S. Kundu, H. K. D. H. Bhadeshia: Scripta Materialia 55 (2006) 779-781.

[22] L. Kestens, R. Decocker, R. Petro: Materials Science Forum 408-412 (2002) 1173-1178.

[23] H. Miyaji, E.-I. Furubayashi: Textures and Microstructure 12 (1990) 189-197.

[24] E.-I. Furubayashi, H. Miyaji, M. Nobuki: Trans. Iron Steel Institute of Japan 27 (1987) 513519 .

[25] R. P. L. Kestens, Y. Houbaert: ISIJ International 43 (2003) 1444-1452.

[26] B. Brückner, G. Gottstein: ISIJ International 41 (2001) 468-477.

[27] P. Bate, B. Hutchinson: Acta Materialia 48 (2000) 3183-3192.

[28] G. Nolze: Zietschrift für Metallkunde 95 (2004) 744-755.

[29] S. Kundu: Transformation strain and crystallographic texture in steels: Ph.D. thesis: University of Cambridge: Cambridge, U. K. (2007).

[30] S. Kundu, K. Hase, H. K. D. H. Bhadeshia: Proceedings of the Royal Society A 463 (2007) 2309-2328.

[31] K. M. Knowles, D. A. Smith, W. A. T. Clark: Scripta Metallurgica 16 (1982) 413-416.

[32] J. W. Christian: Theory of Transformations in Metal and Alloys, Part II: 3rd Edition: Pergamon Press, 2003. 
[33] D. W. Suh, H. N. Han, S. J. Kim: ISIJ International 46 (2006) 341-343.

[34] Y. Higo, F. Lecroisey, T. Mori: Acta Metallurgica 22 (1974) 313-323.

[35] J. R. Patel, M. Cohen: Acta Metallurgica 1 (1953) 531-538.

[36] H. N. Han, D. W. Suh: Acta Materialia 51 (2003) 4907-4917.

[37] H. K. D. H. Bhadeshia, S. A. David, J. M. Vitek, R. W. Reed: Materials Science and Technology 7 (1991) 686-698.

[38] A. Matsuzaki, H. K. D. H. Bhadeshia, H. Harada: Acta Metallurgica and Materialia 42 (1994) 1081-1090.

[39] H. K. D. H. Bhadeshia: Possible effects of stress on steel weld microstructures: in: H. Cerjak, H. K. D. H. Bhadeshia (Eds.), Mathematical Modelling of Weld Phenomena - II: Institute of Materials, London, U.K., 1995: pp. 71-118.

[40] J. W. Stewart, R. C. Thomson, H. K. D. H. Bhadeshia: Journal of Materials Science 29 (1994) 6079-6084.

[41] S. Kundu, H. K. D. H. Bhadeshia: Scripta Materialia 57 (2007) 869-872. 